\title{
Percutaneous Radiofrequency Ablation of Osteoid Osteomas with Use of Real-Time Needle Guidance for Accurate Needle Placement: A Pilot Study
}

\author{
Wendy M. H. Busser • Yvonne L. Hoogeveen • Rene P. H. Veth • \\ H. W. Bart Schreuder • Angelique Balguid · W. KlaasJan Renema • \\ Leo J. SchultzeKool
}

Received: 13 January 2010/Accepted: 28 June 2010/Published online: 29 July 2010

(c) The Author(s) 2010. This article is published with open access at Springerlink.com

\begin{abstract}
Purpose To evaluate the accuracy and technical success of positioning a radiofrequency ablation (RFA) electrode in osteoid osteomas by use of a new real-time needle guidance technology combining cone-beam computed tomography (CT) and fluoroscopy.

Materials and Methods Percutaneous RFA of osteoid osteomas was performed in five patients (median age 18 years), under general anesthesia, with the use of conebeam CT and fluoroscopic guidance for electrode positioning. The outcome parameters were technical success, meaning correct needle placement in the nidus; accuracy defined as the deviation (in $\mathrm{mm}$ ) from the center of the nidus; and clinical outcome at follow-up.

Results In all five cases, positioning was possible within $3 \mathrm{~mm}$ of the determined target location (median nidus size $6.8 \mathrm{~mm}$; range 5-10.2 mm). All procedures were technically successful. All patients were free of pain at clinical follow-up. No complications were observed.

Conclusion Real-time fluoroscopy needle guidance based on cone-beam CT is a useful tool to accurately position radiofrequency needles for minimally invasive treatment of osteoid osteomas.
\end{abstract}

W. M. H. Busser ( $₫)$ · Y. L. Hoogeveen ·

W. K. Renema · L. J. SchultzeKool

Department of Radiology, Radboud University Nijmegen

Medical Centre, PO Box 9101, 6500 HB Nijmegen,

The Netherlands

e-mail: w.busser@rad.umcn.nl

R. P. H. Veth - H. W. B. Schreuder

Department of Orthopedic Surgery, Radboud University

Nijmegen Medical Centre, Nijmegen, The Netherlands

A. Balguid

Philips Healthcare, Best, The Netherlands
Keywords Osteoid osteoma - Radiofrequency ablation · Real-time needle guidance

\section{Introduction}

Osteoid osteoma is a benign skeletal tumor that accounts for approximately $10 \%$ of all benign bone tumors [1,2]. Patients with osteoid osteoma are mostly children or young adults and more frequently male. Although lesions can occur in almost every bone, osteoid osteomas are usually found in the lower extremity with a prevalence for femur and tibia. A striking radiological feature is the radiolucent nidus surrounded by an area of dense osteosclerosis [3]. This nidus has low growth potential and usually has a diameter smaller than $15 \mathrm{~mm}$, which is considered small $[1,3]$. This small size leads to difficulties in visualizing and targeting the nidus.

Invasive treatments include complete surgical resection, percutaneous excision by hollow drills, and percutaneous radiofrequency ablation (RFA). RFA involves the use of thermal coagulation of the nidus to induce necrosis in the osteoid osteoma. Rosenthal et al. [4] compared RFA to surgical treatment. Although equivalent treatment outcome was concluded, RFA was preferred because of shorter hospital stay and shorter recovery time. Various needle guidance approaches have been described as minimally invasive and effective methods for the percutaneous treatment of osteoid osteomas, such as computed tomographic (CT) guidance [1, 2, 5, 6], fluoroscopic guidance [7], or ultrasonographic guidance [8].

Although CT is a good technique to visualize the nidus and its surrounding soft tissue, it has its limitations in needle guidance. Real-time guidance is not possible unless CT-fluoroscopy is used with an associated increase in radiation dose. Furthermore, the planned needle path has to 
be within the imaging plane, which restricts the angle of approach. Especially for procedures requiring a doubleangulated approach, this is a limiting factor.

Real-time needle guidance is possible with fluoroscopy. However, the visualization of the targeted nidus is suboptimal, and the images offer no information about the surrounding soft tissues [7]. Osteoid osteoma is difficult to distinguish on ultrasound, so therefore it is not the modality of choice $[9,10]$.

Limitations of the above-mentioned techniques have resulted in the development of a novel real-time needle guidance technique for tracking and navigation, combining cone-beam CT and fluoroscopic guidance for needle placement in the interventional radiology suite [11]. The purpose of this report is to describe our experience with this guidance technique for accurate positioning of radiofrequency electrodes to ablate small targets, such as osteoid osteomas.

\section{Materials and Methods}

\section{Patients}

RFA was performed on five patients, four male and one female (median age 18 years; range 5-51 years), with osteoid osteoma. The diagnosis was based on severe pain that did not respond to aspirin, and radiological features. Lesions were located in corpus L5 $(n=1)$, acetabulum $(n=1)$, femur $(n=2)$, and tibia $(n=1)$. The median nidus size was $6.8 \mathrm{~mm}$ (range 5-10.2 mm). Patient and nidus characteristics are summarized in Table 1 . In one patient, an earlier unsuccessful attempt was made to access the nidus under conventional CT guidance with a drill.

The study was exempted for approval by the institutional review board because the commercially available system is used in standard clinical practice in our hospital for interventional procedures.

Table 1 Nidus sizes and deviations for all patients

\begin{tabular}{lllll}
\hline Patient no. & Sex & $\begin{array}{l}\text { Age } \\
\text { (years) }\end{array}$ & $\begin{array}{l}\text { Nidus size } \\
(\mathrm{mm})\end{array}$ & $\begin{array}{l}\text { Deviation from nidus } \\
\text { center }(\mathrm{mm})\end{array}$ \\
\hline 1 & $\mathrm{M}$ & 13 & 5 & 0 \\
2 & $\mathrm{M}$ & 50 & 5 & 2.3 \\
3 & $\mathrm{M}$ & 51 & 6.8 & 1.8 \\
4 & $\mathrm{M}$ & 18 & 10.2 & 0.6 \\
5 & $\mathrm{~F}$ & 5 & 9.9 & 0 \\
Median & & 18 & 6.8 & 0.6 \\
\hline
\end{tabular}

Procedure

All procedures were performed with the patient under general anesthesia in the interventional room under aseptic conditions. General anesthesia is necessary because RFA treatment is extremely painful.

To determine the exact location of the nidus, a conebeam CT scan was performed with the Allura Xper FD-20 system (Philips Medical Systems, Best, The Netherlands). Subsequently, the system's XperGuide functionality, needle planning, and navigation software tool, which creates an overlay of live fluoroscopy and 3D soft tissue images (Philips XperCT), was used in the next phases of the procedure. First, the 3D volume reconstructed from the XperCT images was used to plan the needle trajectory from the skin entry point to the center of the nidus (target location). The determined path was projected onto realtime fluoroscopy images together with an overlay of the XperCT images to allow exact needle placement on the patient's skin and constant monitoring and adjustments during needle progression. The software also calculates the position of the X-ray tube to view the entry point of the needle on the skin. This was used to automatically move the gantry and precisely align the 11-gauge introducer (StarBurst Access System; RITA Medical Systems, Mountain View, CA) to the determined path. The progression of the needle into the patient was followed in real time under fluoroscopic guidance after selecting the progression view, which rotates the gantry $90^{\circ}$ perpendicularly to the trajectory. By using the guiding views, the introducer was placed at the bone cortex. Thereafter, the Bonopty Penetration Set or Extended Drill (Radi Medical Systems, Uppsala, Sweden) was used to drill through the cortex towards the nidus. A second XperCT scan was performed to confirm the correct position of the drill. When the nidus was reached, the drill was replaced by the StarBurst SDE radiofrequency electrode (17 gauge, $12 \mathrm{~cm}$; RITA Medical Systems) connected to the radiofrequency generator model 1500 of RITA (power of $50 \mathrm{~W}$ ). Two grounding pads, placed on the patient's upper legs during patient preparation, were used to disperse the heat. The electrode tip was placed as near as possible to the center of the nidus, and its temperature was increased to $85^{\circ} \mathrm{C}$. This temperature was maintained for 4 minutes, ablating the nidus in a sphere with a diameter of $2 \mathrm{~cm}$ to ensure ablation of the entire nidus. After ablation, the electrode and introducer were withdrawn, and a bandage was applied.

The procedure was regarded as technically successful if the electrode was placed within the nidus and the drill had followed the predetermined path toward the planned target. The accuracy of the needle placement is defined as the deviation (in $\mathrm{mm}$ ) from the center of the nidus and the planned path. 
Patients were adminitsered pain medication to control postprocedural pain and were discharged from the hospital on the same day unless the pain was too severe.

According to our clinical practice, clinical follow-up was 2 months post-procedure during an outpatient visit to assess the clinical outcome of the procedure in terms of pain and the need for additional treatments.

\section{Results}

The nidus sizes were 5, 5, 6.8, 9.9, and $10.2 \mathrm{~mm}$ (Fig. 1). The needle was placed within the nidus in all five cases, with a maximum deviation of $2.3 \mathrm{~mm}$ (range $0-2.3 \mathrm{~mm}$; Table 1) from the determined target location (the center of the nidus) indicating technical success (Fig. 2A, B). Each nidus was reached with the radiofrequency electrode at the first attempt. RFA was successfully performed in all five patients.

The intraprocedural XperCT images indicated that for all patients there was no change in body position during the procedure. No complications were observed in any of the patients.

At clinical follow-up, all patients were free of pain and needed no further treatment with nonsteroidal anti-inflammatory drugs, which we regarded as clinical success.

In four of the five patients, the needle trajectories were in the axial plane. For the fifth patient, an oblique trajectory was required to reach the nidus located in L5. In the entry

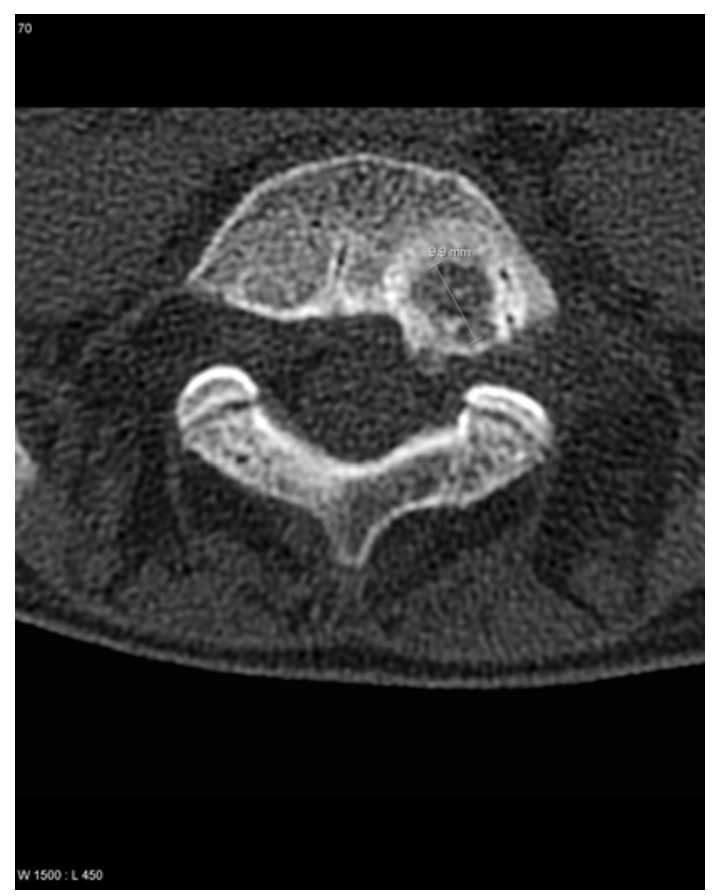

Fig. 1 Computed tomographic image of the osteoid osteoma located in L5 in patient 5, which required a double-angulated approach
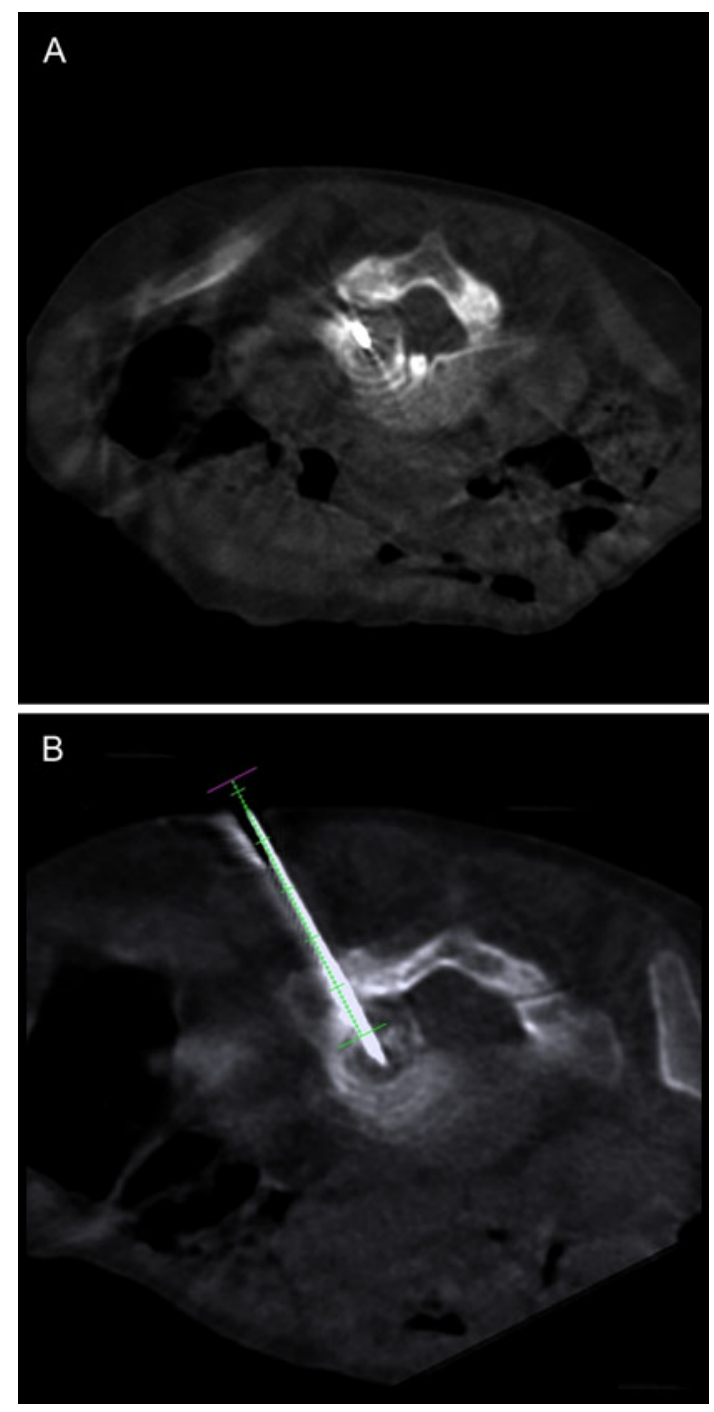

Fig. 2 a XperCT image with needle placed in the nidus located in L5 (axial plane). b XperCT image with the needle in place. The needle has followed the planned double-angulated trajectory into the nidus (rotation $-27^{\circ}$, angulation $58^{\circ}$ )

point view position, the $\mathrm{C}$ arm was rotated $-27^{\circ}$ and angulated $-32^{\circ}$. In the progression view, the $\mathrm{C}$ arm was rotated $-27^{\circ}$ and angulated $58^{\circ}$ (Fig. 2B).

\section{Discussion}

Percutaneous RFA has already been shown to be effective in treating osteoid osteomas. Other authors report initial clinical success rates $(n=12-47)$ of $77-94 \%$ and secondary success rates after repeated radiofrequency treatments of $92-100 \%[5,6,12,13]$. The results of this initial experience of our small series of patients with XperGuide to aid in exact needle placement show an initial clinical success rate of 5 out of 5 . 
Dupuy et al. [14] have shown that RFA in bones has significant effect in pain reduction at follow-up at 1 and 3 months. Our follow-up at 2 months is therefore sufficient to determine clinical success in terms of pain relief.

During needle progression toward a particular target, body position changes can negatively affect precise targeting. This can be minimized as the XperCT scan enables path determination during the procedure. For our patients, who were treated under general anesthesia, no changes in body position were observed between XperCT scans acquired during planning and after needle insertion, thereby allowing accurate localization and targeting of the nidus without the need for additional path realignment.

Overlaying the XperCT images with the predetermined path and real-time fluoroscopy images has several benefits. First, the optimal path is planned by allocating the target and skin entry point so as to avoid important anatomical structures such as vessels and nerves. This allows confident and continuous adjustments to the introducer so that the correct path is followed. The entry point view and progression views perpendicular to the path are automatically calculated by the system, thereby attaining the optimal gantry position. The views obtained with the software allow monitoring of drill progression in relation to the predetermined path and the underlying soft tissue, which possibly has the advantage of limiting unnecessary tissue damage and damage to critical structures.

In our experience, XperGuide also provides the benefit of being able to access the nidus from any direction. The path can be planned in any direction through the entire three-dimensional volume for either the shortest or safest access. When CT is used for needle guidance, the trajectory is limited to the axial plane or the scanning plane with the gantry at the maximum tilt of approximately $25^{\circ}-30^{\circ}$. In our fifth patient, an axial path would not have provided access to the nidus located in L5 without damaging nerve roots. A gantry angulation of $-32^{\circ}$ was used for the entry point view in this particular case and $58^{\circ}$ for the progression view.

To fully assess the additional value of XperGuide in treatments such as RFAs, further extended studies are required.

The results of our study indicate that the combined needle guidance technology is a useful tool for precise needle positioning in small osteoid osteomas for treatment with RFA.
Conflicts of interest A. Balguid is a salaried employee of Philips and has no commercial interest in the company. The other authors declare that they have no conflict of interest.

Open Access This article is distributed under the terms of the Creative Commons Attribution Noncommercial License which permits any noncommercial use, distribution, and reproduction in any medium, provided the original author(s) and source are credited.

\section{References}

1. Rosenthal DI, Hornicek FJ, Torriani M et al (2003) Osteoid osteomas: percutaneous treatment with radiofrequency energy. Radiology 229:171-175

2. Barei DP, Moreau G, Scarborough MT et al (2000) Percutaneous radiofrequency ablation of osteoid osteoma. Clin Orthop Relat Res 373:115-124

3. Kransdorf MJ, Stull MA, Gilkey FW et al (1991) Osteoid osteoma. Radiographics 11:671-696

4. Rosenthal DI, Hornicek FJ, Wolfe MW et al (1998) Percutaneous radiofrequency coagulation of osteoid osteoma compared with operative treatment. J Bone Joint Surg Am 80:815-821

5. Rosenthal DI, Alexander A, Rosenberg AE et al (1992) Ablation of osteoid osteomas with a percutaneously placed electrode: a new procedure. Radiology 183:29-33

6. Lindner N, Ozaki T, Roedl R et al (2001) Percutaneous radiofrequency ablation in osteoid osteoma. J Bone Joint Surg $\mathrm{Br}$ 83:391-396

7. Graham HK, Laverick MD, Cosgrove AP et al. (1993) Minimally invasive surgery for osteoid osteoma of the proximal femur. J Bone Joint Surg Br 75B:115-118

8. Mehdizade A, Danon M, Ellis S et al (2006) Use of ultrasonographic guidance for needle localization of osteoid osteoma of the capitates. HSS J 2:176-180

9. Ebrahim SF, Jacobson JA, Lin J et al (2000) Intraarticular osteoid osteoma: sonographic findings in three patients with radiographic, CT, and MR imaging correlation. AJR Am J Roentgenol 177:1391-1395

10. Gil S, Marco SF, Arenas J et al (1999) Doppler duplex color localization of osteoid osteomas. Skelet Radiol 28:107-110

11. Racadio JM, Babic D, Homan R et al (2007) Live 3D guidance in the interventional radiology suite. AJR Am J Roentgenol 189:W357-W364

12. Woertler K, Vestring M, Boettner F et al (2001) Osteoid osteoma: CT-guided percutaneous radiofrequency ablation and follow-up in 47 patients. J Vasc Interv Radiol 12:717-722

13. Mahnken AH, Tacke JA, Wildberger JE et al (2006) Radiofrequency ablation of osteoid osteoma: initial results with a bipolar ablation device. J Vasc Interv Radiol 17:1465-1470

14. Dupuy DE, Liu D, Hartfeil D et al (2010) Percutaneous radiofrequency ablation of painful osseous metastases. Cancer 116:989-997 